\title{
Adaptive Line Size Cache for Irregular References on Cell Multicore Processor
}

\author{
Qian Cao, Chongchong Zhao*, Junxiu Chen, \\ Yunxing Zhang, and Yi Chen \\ University of Science and Technology Beijing, \\ 100083 Beijing, China \\ caoqian125@126.com
}

\begin{abstract}
Software cache promises to achieve programmability on Cell processor. However, irregular references couldn't achieve a considerable performance improvement since the cache line is always set to a specific size. In this paper, we propose an adaptive cache line prefetching strategy which continuously adjusts cache line size during application execution. Therefore, the transferred data is decreased significantly. Moreover, a corresponding software cache adaptive line size cache is designed. It introduces a hybrid Tag Entry Arrays, with each mapping to a different line size. It's a hierarchical design in that the misshandler is not invoked immediately when an address is a miss in the short line Tag Entry Array. Instead, the long line Tag Entry Array is checked first, which significantly increases the hit rate. Evaluations indicate that improvement due to the adaptive cache line strategy translates into 3.29 to 5.73 speedups compared to the traditional software cache approach.
\end{abstract}

Keywords: Adaptive, Software cache, Irregular reference, Cell processor.

\section{Introduction}

Irregular application is widely used in scientific computing, which exposes unclear aliasing and data dependence information. Such applications are frequently seen in reservoir numerical simulation, molecular dynamics, etc.

Heterogeneous multicore is an area and energy efficient architecture to improve performance for domain-specific applications. The Cell processor is a representative heterogeneous multicore, which comprises a conventional Power Processor Element (PPE) that controls eight simple Synergistic Processing Elements (SPEs), as illustrated in Figure 1. PPE has two levels of cache that are coherent with the globally memory, while SPEs don't have cache but each has 256KB of local store. PPE can access main memory directly while SPE only operates directly on its local store and works as an accelerator. Software cache is a common approach to automatically handle data transfers for irregular reference, providing the user with a transparent view of the memory architecture.

\footnotetext{
* Corresponding author.
} 
There has been substantial research [1-6] on software cache specifically for Cell processor. Eichenberger et al. [1] propose a compiler-controlled software cache. It's a traditional 4-way set-associative cache implemented in software. It adopts the LRU policy and SIMD mode to look up for a match among the four tags in a set.

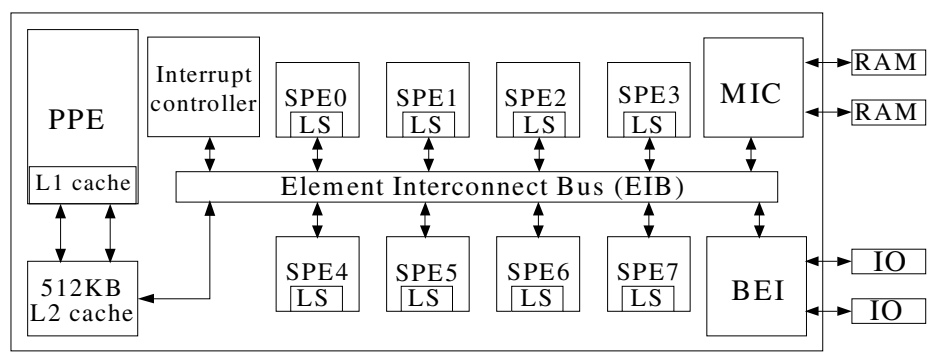

Fig. 1. Cell Architecture

Balart et al. [2] demonstrate a software cache for Cell which adopts hashed list for lookup and allows for full associative. This design enables a user to determine code regions guaranteed not to have any cache conflicts. In such a region, the user can reorder lookup and misshandler operations, so communication and computation can be efficiently overlapped. The strategy usually performs well for specific loops containing few cache accesses with high temporal locality, but it introduces a large implementation overhead for the general applications.

The COMIC runtime system proposed by Lee et al. [3] provides the application with an illusion of a shared memory, in which the PPE and the SPEs can access the shared data. The management of synchronization and coherence is centralized in the PPE and the release consistency is achieved by software cache.

A hybrid access-specific software cache is presented by Marc Gonzalez et al. [4, 5]. It classifies memory accesses into high locality and irregular, and the corresponding high locality cache and the transactional cache are designed. The former applies the write-back mechanism while the latter supports the write-through policy. Its motivation is similar to the direct buffer plus software cache approach.

Chen et al. [6] propose an integrated software cache and direct buffer approach so as to efficiently execute the loops that include both references. Their solution provides compile time analysis and runtime support to minimize the coherency operations.

The software caches usually suffer from poor performance, especially when the irregular reference is encountered. The solutions above always set the cache line to a specific size, which introduces the reduction of data transfers and increases the memory bandwidth overhead. The cache design with adaptive line size could obviously improve the irregular application performance. There are some proposals $[7,8]$ for the hardware adaptive cache line solutions. But the SPE on Cell has no hardware cache, so we focus on adaptive cache line designs implemented in software.

To the best of our knowledge, the adaptive cache line scheme proposed by Sangmin Seo et al. [9] is the only strategy which continuously adjusts the cache line on Cell processor. Their design is called extend set-index cache (ESC), which is based on 
4-way set-associative cache. Nevertheless, the number of TEs could be greater than the number of cache lines. The adaptive strategy in ESC utilizes the runtime to adapt to characteristics specific to the loop considering that the loops are invoked many times. But their strategy is applied to parallel loops only, and it isn't sensitive to variation across iteration of the loop. Additionally, its storage overhead is large.

In this paper, we propose an adaptive cache line size strategy, which adaptively adjusts cache line size according to the characteristics specific to the irregular reference. The solution gathers the addresses accessed by the irregular reference and divides them into long line addresses (long addresses) and short line addresses (short addresses). The algorithm adaptively chooses the optimal cache line size, regardless of how many times the loop is invoked.

Moreover, a corresponding software cache design - adaptive line size cache (ALSC) is presented. It is based on the 4-way set-associative cache (4WC) and adopts a hybrid Tag Entry Arrays, a long line Tag Entry Array and a short line Tag Entry Array, with each mapping to a different line size. The operations to the long line Tag Entry Array is the same as the traditional 4WC, but when a miss occurs in the short line Tag Entry Array, the misshandler is not invoked at once. Instead, the long line Tag Entry Array is checked. So the miss rate is significantly decreased.

In order to implement cache replacement policy for cache design with multiple line sizes, we present a novel LRU policy - IndAlign_LRU. It adopts a link array, with each link mapping to one set in the long line Tag Entry Array and two successive sets in the short line Tag Entry Array. The data field of the link node stores the cache line index. IndAlign_LRU policy is implemented by moving nodes to the link head or tail.

The experimental results show that our approach obtains speedup factors from 3.29 to 5.73 compared to the traditional software cache scheme with specific line size. Moreover, it significantly reduces the miss rate and the total transferred data size. Additionally, the adaptive approach we proposed shows good scalability.

The rest of the paper is organized as follows. The adaptive software cache line algorithm is presented in Section 2. Section 3 describes the ALSC design. The ALSC operational model is presented in Section 4. Section 5 evaluates our adaptive approach. The last section concludes the paper.

\section{The Adaptive Software Cache Line Algorithm}

The adaptive cache line prefetching scheme, which is based on our previous work [15], consists of four steps. A loop with normalized boundaries is extracted from CG in the NAS benchmark suite for a clear explanation, as illustrated in Figure 2a. And the adaptive strategy is shown in Figure $2 b$.

The first step is to initialize the cache lines. For the sake of simplicity, two cache lines, 128B and 256B, are introduced.

The second step is to divide the addresses into long and short addresses. Our adaptive algorithm is applied to each iteration range. We propose a dynamic address collecting solution, which means the address collecting is stopped when the first set conflict is encountered. 




a. Simplified original code

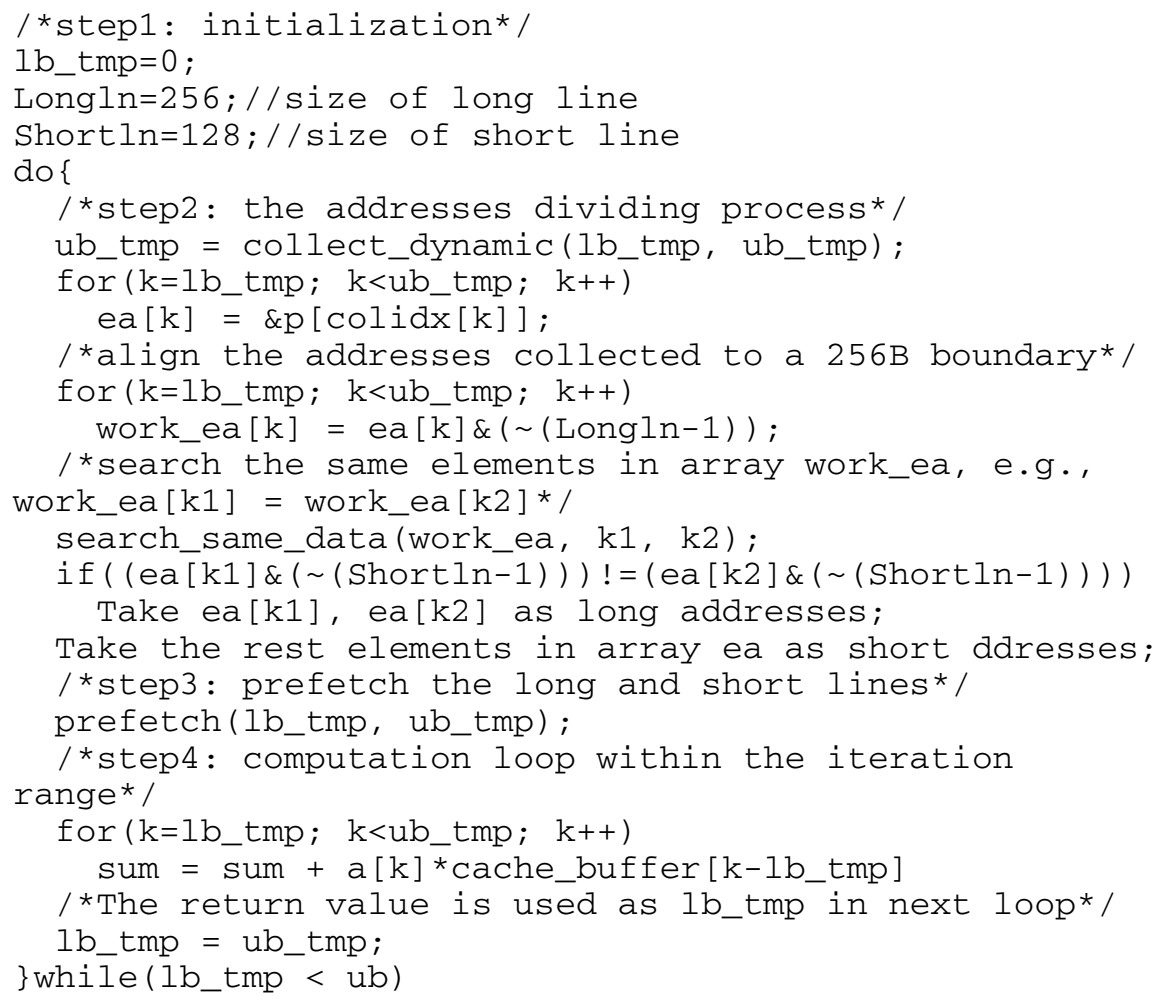

b. After the adaptive software cache line strategy

Fig. 2. Example for adaptive cache line size algorithm

To explain it clearly, an example is shown in Figure 3. We assume the following:

- There are seven memory requests with addresses ranging from $a_{1}$ to $a_{7}$ in an iteration range and they are all mapped to the addresses in the range of $256 \mathrm{~N}$ $(256(\mathrm{~N}+2)-1))$.

- The data located in addresses from $256 \mathrm{~N}$ to $(256(\mathrm{~N}+1)-1)$ is represented $L_{\mathrm{i}}$, and the former $128 \mathrm{~B}$ is represented by $L_{\mathrm{i}-1}$ while the latter $128 \mathrm{~B}$ is expressed as $L_{\mathrm{i}-2}$. $L_{\mathrm{i}-1}$ and $L_{\mathrm{i}-2}$ are "adjacent" lines.

- The data located in addresses from $256(\mathrm{~N}+1)$ to $(256(\mathrm{~N}+2)-1)$ is represented $L_{\mathrm{j}}$, and the former $128 \mathrm{~B}$ is represented by $L_{\mathrm{j}-1}$ while the latter $128 \mathrm{~B}$ is expressed as $L_{\mathrm{j}-2} . L_{\mathrm{j}-1}$ and $L_{\mathrm{j}-2}$ are "adjacent" lines.

All the seven addresses are taken as short addresses initially, as illustrated in Figure $3 \mathrm{a}$. The addresses $a_{1}$ and $a_{3}$ are mapped to the "adjacent" lines. If a short line is fetched from the memory, two DMA operations which respectively fetch the $L_{\mathrm{i}-1}$ and 
the $L_{\mathrm{i}-2}$ are required. So our adaptive strategy makes the two short lines $L_{\mathrm{i}-1}$ and the $L_{\mathrm{i}-2}$ merged into a long line $L_{\mathrm{i}}$. And the addresses $a_{1}$ and $a_{3}$ are taken as long addresses. Obviously, the data required can be obtained in one DMA. Analogically, addresses $a_{2}$, $a_{4}, a_{5}$ are regarded as long addresses.

The data located in addresses $a_{6}$ and $a_{7}$ is in $L_{\mathrm{j}-2}$, and there's no memory requirement in its "adjacent" line $L_{\mathrm{j}-1}$, so the address $a_{6}$ and $a_{7}$ are both taken as short line addresses.

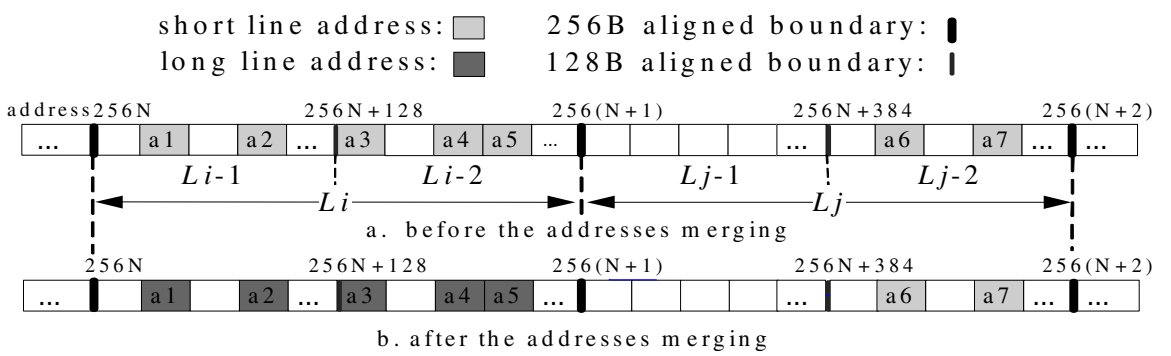

Fig. 3. Example for addresses merging

The third step is to adaptively prefetch the long and short lines from lower bound $l b \_t m p$ up to upper bound $u b \_t m p$.

The last step is the computation loop, which performs the computation.

\section{The Adaptive Line Size Cache Structure}

We design a software cache which corresponds to the adaptive cache line algorithm. For the sake of simplicity, we describe the cache including only two kinds of lines, $128 \mathrm{~B}$ and 256B. It's composed of the following structures, as depicted in Figure 4.

The Cache Storage is set to be $64 \mathrm{~KB}$ in this paper.

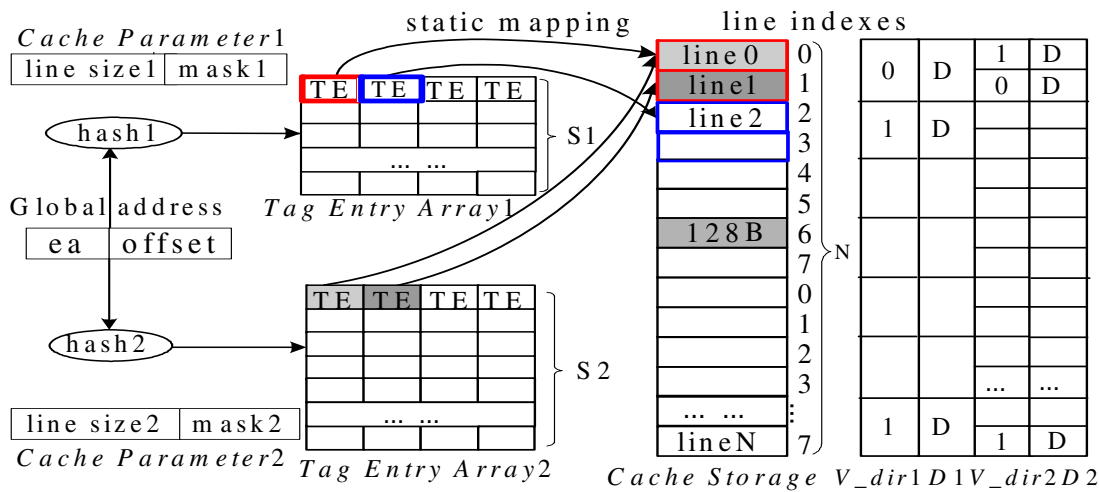

Fig. 4. The ALSC structure 
The Cache Parameter $_{1}$ includes two components, the mask $k_{1}$ and the line size $e_{1}\left(L_{1}\right)$. $L_{1}$ is set to be $256 \mathrm{~B}$, so the number of the long line $\left(n \_L\right)$ is $256(64 \mathrm{~KB} / 256 \mathrm{~B})$.

The Tag Entry Array ${ }_{1}$ is a long address tag lookup table which is composed of $S_{1}$ $\left(S_{1}=n \_L / 4=64\right)$ sets. Each TE statically maps to a $256 \mathrm{~B}$ long line.

The Cache Parameter Pa $_{2}$ is similar to the Cache Parameter ${ }_{1}$. The line size Pa $_{2}\left(L_{2}\right)$ is set to be $128 \mathrm{~B}$ and the number of the short line $\left(n \_S\right)$ is 512 .

In Tag Entry Array 2 , each tag statically maps to a short line.

Every set in the Tag Entry Array ${ }_{1}$ maps to 8 successive short cache lines. The short lines are orderly numbered $0,1,2, \ldots, 7,0,1,2, \ldots, 7$, which are the line indexes.

In order to implement LRU replacement policy among multiple line sizes, we extend the traditional LRU replacement policy and present IndAlign_LRU, which adopts an Index Link Array. Its initial information and the mapping relationship between the Index Link Array and the cache lines are illustrated in Figure 5.

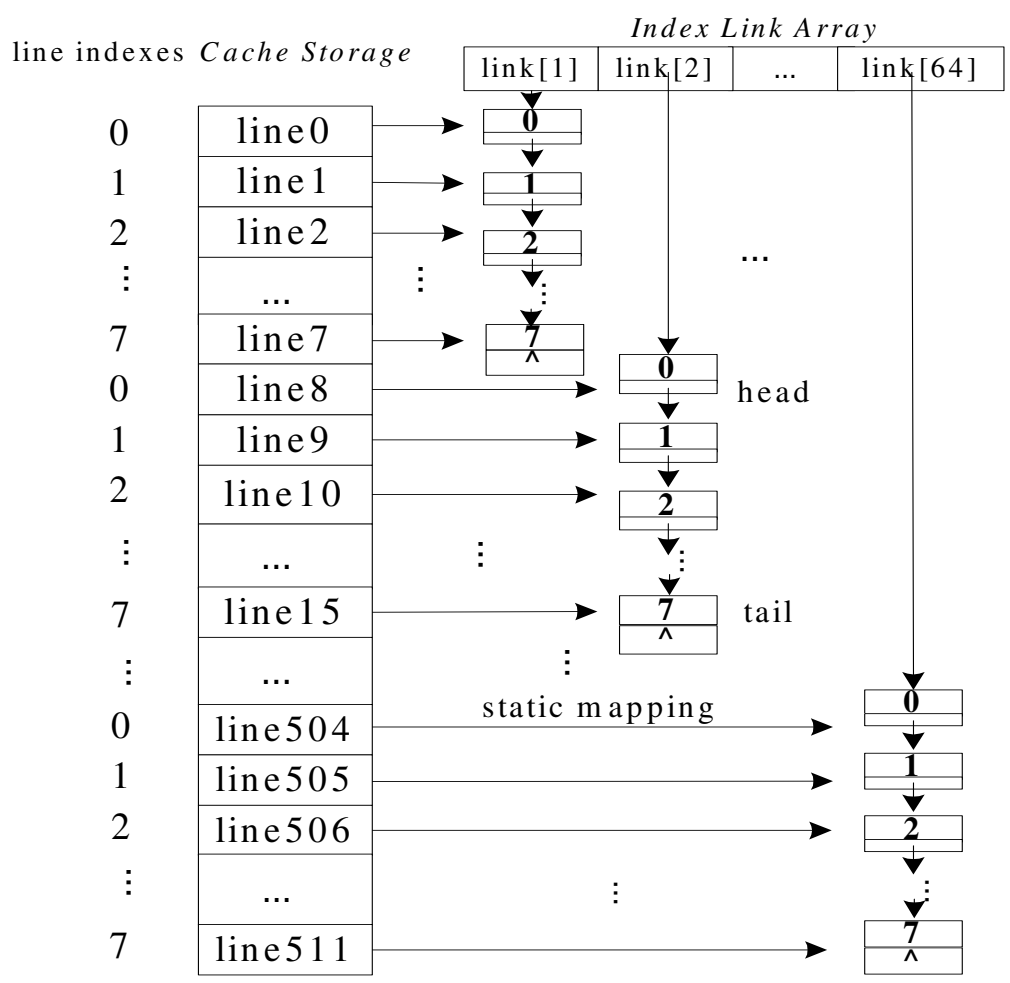

Fig. 5. The mapping relationship between the Index Link Array and cache lines

The Index Link Array is a link array which has $S_{1}$ links. Each link is mapped to one set in the Tag Entry Array ${ }_{1}$ and two successive sets in the Tag Entry Array 2 . Each link contains 8 nodes, with each node having a data field to store the line index. The data filed of link head stores the index of the cache line which is the earliest accessed while the data field of the link tail stores the index of the line which is the latest accessed. The cache line activity is recorded by moving nodes to the head or the tail. 
The $V \_d i r_{1}$ and $V \_d i r_{2}$ record the valid bits and the $D_{1}$ and $D_{2}$ store the dirty bytes.

The cache architecture we propose is a hierarchy design. Figure 6 shows set masks of the long line address and short line address. Both the numbers of set in Tag Entry Array $_{1}$ and Tag Entry Array 2 are powers of 2, so a bit-wise AND operation instead of the hash function is used to improve performance, as shown in (1).

$$
\text { SetID }=(\text { ea \& SetMask })>>\text { N_bits }
$$

Where SetID is the number of the set, SetMask is the set mask and $2^{\mathrm{N} \_ \text {bits }}$ equals the corresponding cache line size.

Because the number of the set in Tag Entry Array is $_{1} 64$ and the line size ${ }_{1}$ is $256 \mathrm{~B}$, the SetID in Tag Entry Array ${ }_{1}$ is decided by the successive 6 bits, ranging from 8th to the 13th bit. Correspondingly, the SetID in Tag Entry Array ${ }_{2}$ is decided by the bits from 7 th to the 13 th. When the cache receives a memory request with a global address $e a$, if it's a miss in the Tag Entry Array ${ }_{2}$, it may be a hit in the Tag Entry Array ${ }_{1}$. Since the number of the bits which decide the SetID is one bit less.

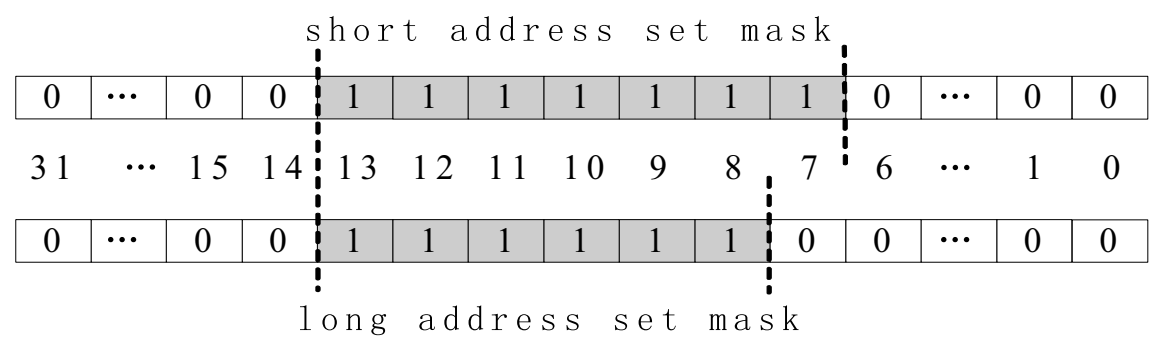

Fig. 6. Set masks of the long line address and short line address

\section{The ALSC Operational Model}

The simple ALSC operational flowchart is shown in Figure. 7.

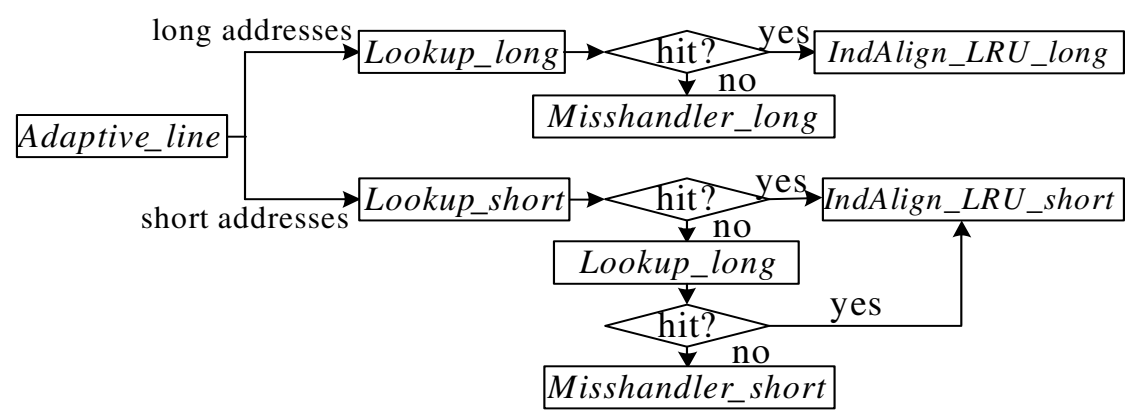

Fig. 7. The ALSC operational flowchart 


\subsection{Lookup_long, Lookup_short, IndAlign_LRU_long, IndAlign_LRU_short}

Both Lookup_long and Lookup_short are the same as the traditional 4WC. IndAlign_LRU_long and IndAlign_LRU_short are invoked respectively when the long address hit and short address hit occur.

Suppose a hit of the long address is encountered and the matching set and way is set_L and hit_index_L respectively, the set_Lth link nodes whose data fields are $\left(2 * h i t \_i n d e x \_L\right)$ and $\left(2 * h i t \_i n d e x \_L+1\right)$ moves to the link tail. This operation is called IndAlign_LRU_long. Figure 8a illustrates the operation when hit_index_L is 0 .

Suppose a short line address comes subsequently and the matching set and way is set_S and hit_index_S respectively, the set_Lth set in the Tag Entry Array ${ }_{1}$ and the set_Sth set in the Tag Entry Array 2 are both mapped to the set_Lth link if the equation $\left(\right.$ set_L $\left.L^{*}=s e t \_S\right)$ or $\left(s e t \_L * 2+1=s e t \_S\right)$ is satisfied.

If the former or the latter is satisfied, the node whose data field is hit_index_S or (hit_index_S+4) moves to the link tail, respectively. The two cases are illustrated in Figure $8 \mathrm{~b}$ and Figure $8 \mathrm{c}$ respectively. The operations are called IndAlign_LRU_short.

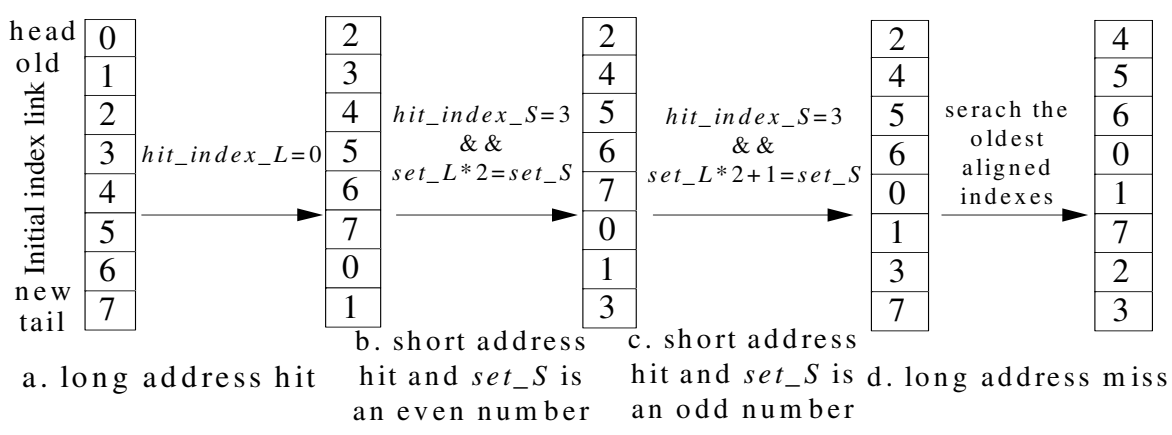

Fig. 8. Examples for the operations in ALSC

\subsection{Misshandler_long}

For a long address, the function IndAlign(index_L) is defined as follows. If index_L is an even number, IndAlign(index_L) is defined as

$$
\text { IndAlign }(\text { index_L })=\text { index_L And }(\text { index_L }+1) \text {. }
$$

If index_L is an odd number, IndAlign(index_L) is defined as

$$
\operatorname{IndAlign}(\text { index_L })=(\text { index_L }-1) \text { And index_L. }
$$

When a long address which is mapped to the set_Lth set arrives subsequently and a miss occurs, Misshandler_long is invoked, which includes the following phases: 
Choosing the oldest lines to be the victim. We first get the oldest indexes, index_old from the set_Lth link head. The two successive short lines whose indexes are IndAlign(index_old) (as defined in (2)(3)) are chosen to be the victims.

Writing back the dirty bytes and setting the corresponding V_dir bits.

Fetching the long line required from the main memory and filling the corresponding DE. Moreover, the nodes whose data fields are IndAlign(index_old) moves to the tail of the set_Lth link, as shown in Figure 8d.

\subsection{Misshandler_short}

For a short address which is mapped to the set_Sth set, IndAlign(index_S) is defined as follows. If set_S is an even number, IndAlign(index_S) is defined as

$$
\operatorname{IndAlign}(\text { index_S })=\operatorname{oldest}(0,1,2,3) \text {. }
$$

If $s e t \_S$ is an odd number, IndAlign(index_S) is defined as

$$
\operatorname{IndAlign}(\text { index_S })=\operatorname{oldest}(4,5,6,7) \text {. }
$$

The oldest(ind1, ind2, ind3, ind4) means the index of the line which is the earliest accessed, with the four parameters in parenthesis denoting the line indexes.

If a short address, which is mapped to the set_Sth $($ set_S $/ 2=$ set_L $)$ set in the Tag

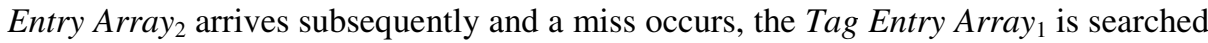
first. If there's a valid matching, the operation is similar to IndAlign_LRU_short. Otherwise, Misshandler_short is called. A simplified ALSC design is shown in Figure 9. We assume the address is mapped to the set_Lth link.

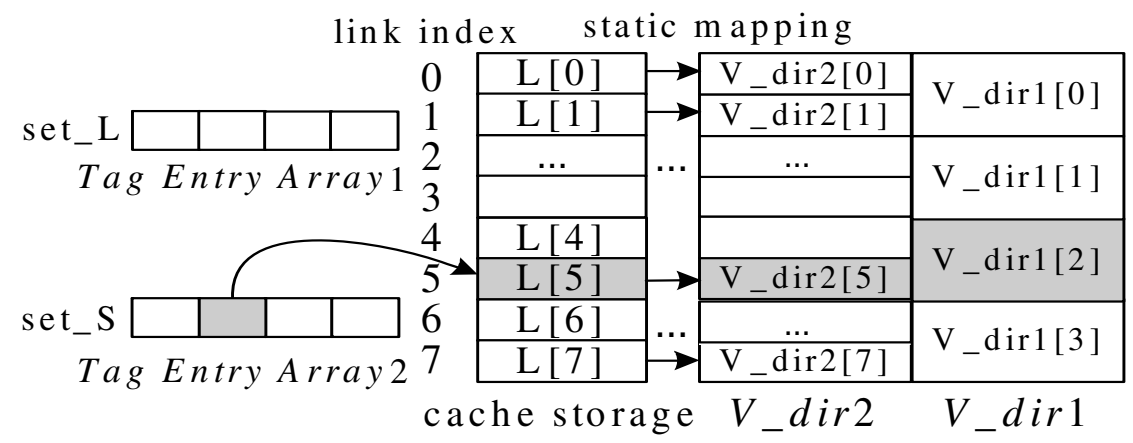

Fig. 9. A simplified ALSC structure 
Choosing the oldest index (index_old) from the set_Lth link according to (4) or (5) after judging the parity of set_S. We assume the index_old is 5 .

Checking the $V \_$dir arrays and writing back the dirty bytes. The $V \_d i r_{1}[2]$, which is mapped by the index_old should be checked first. If it's valid, the dirty bytes in $L[4]$ and $L[5]$ should be written back. Meanwhile, the $V \_d i r_{1}[2]$ is set to be 0 . Otherwise, if it's invalid, the $V \_d i r_{2}[5]$ is checked. If it's valid, the dirty data in $L[5]$ is written back.

Fetching the required short line from the memory, filling the corresponding DE, and setting V_dir 2 [5] to valid. Finally, the node whose data field is 5 moves to the link tail.

\section{Evaluation}

\subsection{Evaluation Environments}

The experiment is conducted on a Cell BE blade [10] with two Cell processors running at $3.2 \mathrm{GHz}$ with $1 \mathrm{~GB}$ of system memory. In this experiment, the programs are bound to one Cell processor to avoid the NUMA effect.

The performance is measured with Sparse matrix-vector (SpMV) multiplication and IS, CG, FT, MG from NAS parallel benchmarks [11]. The sparse matrix epb1.mtx is a $14734 * 14734$ symmetric matrix, which is obtained from the University of Florida Sparse Matrix Collection [12]. The benchmarks IS, CG, FT, MG are tested with CLASS A. The sequential regions are executed on the PPE while the iterations in the parallel loops are distributed among the available SPEs. The system runs Fedora9 (Linux Kernel 2.6.25-14). Our programs are compiled in the Cell SDK3.1.

\subsection{Execution Speed}

In this section, we evaluate four software cache configurations. The first one is a traditional software cache design implementing a 4-way set-associative cache. This cache design is with $64 \mathrm{~KB}$ storage and 128-byte cache lines. It's referred to TRADITIONAL. The second cache configuration is ESC [9]. It is an alternative to implement the adaptive software cache line on Cell processor, so we compare it with our scheme. The last configuration is our adaptive software line size cache, which adopts a $64 \mathrm{~KB}$ cache storage. We refer to this configuration as ADAPTIVE. The last two configurations adaptively choose a cache line size among 128B, 256B, 512B and 1024B during application execution.

Figure 10 illustrates the normalized speedup of different applications, and the baseline is the execution speed of TRADITIONAL. On the whole, our adaptive cache line strategy combined with the optimized cache structure performs better than the other two cache designs. 
We first compare our adaptive cache line solution with the ESC. Obviously, our adaptive cache solution achieves noticeable performance improvements. It mainly results from the following factors:

First, the adaptive algorithm in ESC is applied to the parallel loop only, but it isn't sensitive to variation across iterations of the loop. Our adaptive approach could be applied to not only the parallel loop but also the iteration ranges, so it could choose the optimal cache line size more precisely.

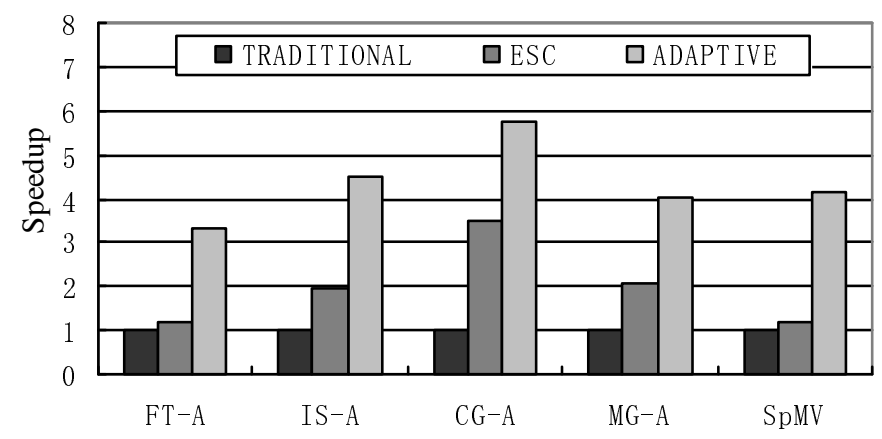

Fig. 10. Normalized speedup

Second, the algorithm in the ESC sometimes might choose a cache line size which is not the optimal. An example is given in Figure 11. There are five different cache line sizes, $L S_{0}$ to $L S_{4}$, with the size increasing. Suppose that their performance levels are $1,3,2,4$, 0 , with each higher level corresponding to the better performance. Obviously, $L S_{3}$ is the optimal line size. The adaptive execution in ESC is as follows.

\begin{tabular}{|c|c|c|c|c|c|}
\hline cache line size & $L S_{0}$ & $L S_{1}$ & $L S_{2}$ & $L S_{3}$ & $L S_{4}$ \\
\hline erformance 1 & 1 & 3 & 2 & 4 & $\begin{array}{c}0 \\
\text { (worst) }\end{array}$ \\
\hline T P I & $T P_{3}$ & $T P I_{2}$ & $T P I_{1}$ & $\mathrm{TPI}_{4}$ & $T P I_{5}$ \\
\hline
\end{tabular}

Fig. 11. An example of the adaptive algorithm in ESC

When the loop is invoked the first time, it's executed with the initial line size, $L S_{2}$. And the time per iteration (TPI) is $T P I_{1}$. When the loop is invoked the next time, it executed with a shorter line, $L S_{1}$. The corresponding TPI is $T P I_{2} . T P I_{1}$ must be greater than $T P I_{2}$ according to the performance level, so a shorter line $L S_{0}$ is chosen and the corresponding $\mathrm{TPI}_{3}$ is measured in the next loop invocation. Obviously, $T P I_{3}$ is greater than $T P I_{2}$, so $L S_{1}$ is chosen to be the optimal. Nevertheless, $L S_{3}$ is the optimal line size according to the performance level. This results from the following two reasons. 
- Though $T P I_{1}$ is greater than $T P I_{2}$ in the above algorithm, it's not necessary the case that a shorter line performs better for the loop, as shown in Figure 11.

- The adaptive strategy in ESC depends on many factors, for example, the initial cache lines size, choosing which line (a longer or a shorter line) in the second invocation of the loop.

Third, the loop has to be invoked many times to compare TPIs in the ESC strategy, which greatly degrades the performance. Additionally, a certain loop may be invoked once in some applications, so the adaptive algorithm in ESC doesn't work well in such cases. The loop in SpMV is invoked only once, and not surprisingly, the ESC has only a slight performance improvement compared with the traditional approach.

Then we compare the ADAPTIVE with the TRADITIONAL configuration. We achieve speedup factors of 3.29 for FT, 4.53 for IS, 5.73 for CG, 4.01 for MG and 4.12 for SpMV. The benchmarks which are sensitive to the cache line size and are dominated by irregular references benefit more from the ADAPTIVE configuration. $\mathrm{CG}$ is such an application, so it achieves a significant performance improvement. Though IS isn't sensitive to the cache line size, it is dominated by irregular memory references and thus it exposes a high miss rate. Not surprisingly, it obtains a significant speedup from our adaptive prefetching scheme.

\subsection{Transferred Data Size}

If the transferred data size of the cache design with $128 \mathrm{~B}$ is the baseline, the transferred data size of cache line design with $128 \mathrm{~B}, 256 \mathrm{~B}, 512 \mathrm{~B}$ and $1024 \mathrm{~B}$ is illustrated in Figure 12.

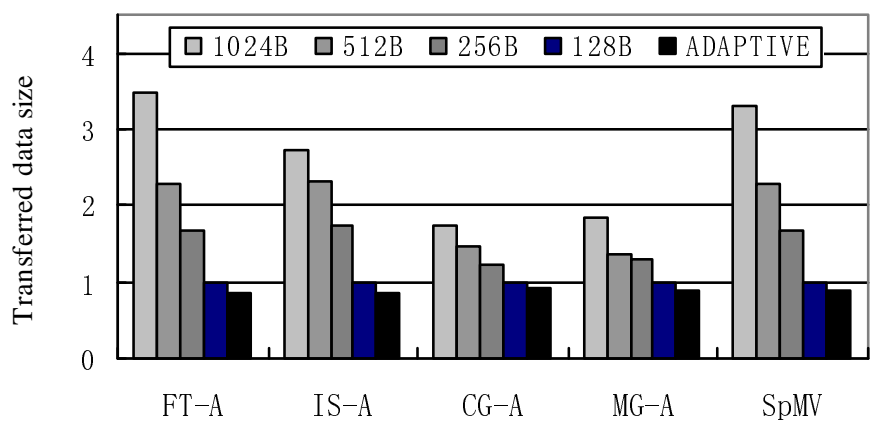

Fig. 12. Normalized transferred data size

Obviously, the transferred data of the ALSC is less than the other cache designs, especially compared with the 1024B line design. Because even though only a few bytes are needed, the whole 1024B line is transferred if the cache line is set to be 1024B. The ALSC outperforms designs with the fixed short cache line because the latter immediately transfers data when a miss occurs. Nevertheless, the ALSC first checks the long line Tag Entry Array. If it's a hit, there is no need to transfer data. 


\subsection{Scalability}

Figure 13 presents the scalability of our approach. All the benchmarks, except IS, show good scalability from 1 to 8 threads, with speedup more than 6 on 8 SPEs. The main reason is that every thread executes in an exclusive SPE. IS doesn't scale up well because it contains some computations in either master execution or critical codes. Those computations are executed sequentially. Therefore, its speedup of 8 SPEs is only about 3.8 .

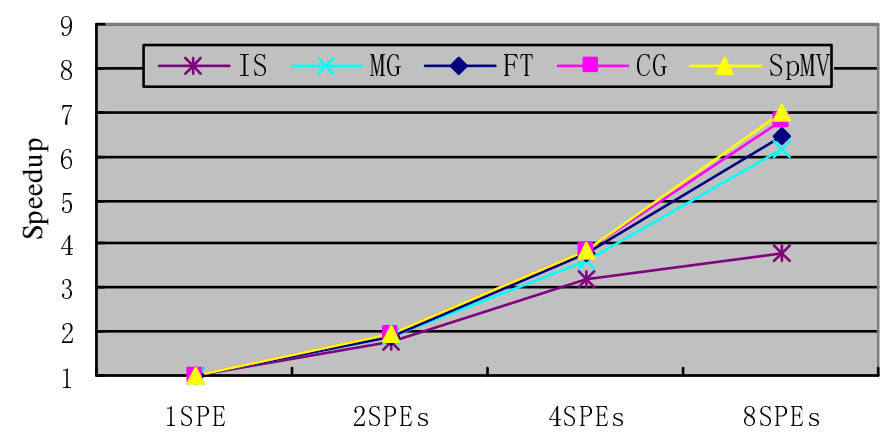

Fig. 13. Scalability of the adaptive approach on Cell processor

\subsection{Storage Overhead}

Table 1 depicts the extra storage overhead except the storage for $N$ cache line. FAC and 4WC denotes the fully associative and 4-way set-associative cache, respectively.

To formally describe the extra storage overhead we assume as followed:

- The number of the short line is $N$, and the number of set in $4 \mathrm{WC}$ is $S(S=N / 4)$.

- $S^{\prime}$ is the number of sets in ESC, which is four times the closest power of 2 to $S$.

- The tags are 4-byte integers while dirty and valid bits are one byte.

- All the caches introduce fetch buffers. And every TE needs a line index. The FAC and the ESC both need a field to record the global address.

Table 1. Extra storage overhead (in bytes)

\begin{tabular}{|c|c|c|c|c|}
\hline Heading level & Example & $4 \mathrm{WC}$ & ESC & ALSC \\
\hline TE & tag+line index $=8$ & tag+line index $=8$ & $10 \mathrm{~N}$ & tag+line index $=8$ \\
\hline TE Array & $\mathrm{TE} * \mathrm{~N}=8 \mathrm{~N}$ & $(4 * \mathrm{TE}) * \mathrm{~S}=8 \mathrm{~N}$ & $(4 * \mathrm{TE}) * \mathrm{~S}^{\prime}=32 \mathrm{~S}^{\prime}$ & $(4 * \mathrm{TE}) * 3 \mathrm{~S} / 2=12 \mathrm{~N}$ \\
\hline LTE & $V+D+\operatorname{tag}=6$ & $\mathrm{~V}+\mathrm{D}=2$ & $\mathrm{~V}+\mathrm{D}+\mathrm{tag}=6$ & $(\mathrm{~V}+\mathrm{D})+(\mathrm{V}+\mathrm{D}) / 2=3$ \\
\hline Cache line table & LTE $* N=6 \mathrm{~N}$ & LTE $* N=2 \mathrm{~N}$ & LTE $* N=6 \mathrm{~N}$ & $\mathrm{LTE}^{*} \mathrm{~N}=3 \mathrm{~N}$ \\
\hline Total Size & $14 \mathrm{~N}$ & $10 \mathrm{~N}$ & $32 \mathrm{~S}^{\prime}+6 \mathrm{~N}$ & $15 \mathrm{~N}$ \\
\hline
\end{tabular}

Suppose that the whole cache size is $64 \mathrm{~K}$, the total storage overhead is listed in Table 2. The ALSC storage overhead is a little more than that of the FAC and 4WC and much less than that of the ESC. 
Table 2. Extra storage overhead with a $64 \mathrm{~KB}$ cache (in bytes)

\begin{tabular}{cccccc}
\hline Line size & Number of lines & FAC & 4WC & ESC & ALSC \\
\hline 128B & 512 & 7168 & 5120 & 19456 & 7680 \\
512B & 128 & 1792 & 1280 & 4864 & 1920 \\
1KB & 64 & 896 & 640 & 2432 & 960 \\
4KB & 16 & 224 & 160 & 608 & 240 \\
\hline
\end{tabular}

\section{Conclusions and Future Works}

We present an algorithm which adaptively adjusts the software cache line for irregular reference on Cell processor. Moreover, a corresponding software cache design is proposed, which significantly improves the hit rate and decreases the reduction of data transfers. The evaluation results indicate that our strategy achieves the speedup factor from 3.29 to 5.73 compared with the traditional software cache approach. Additionally, the adaptive strategy shows good scalability.

Acknowledgments. The research is partially supported by the Hi-Tech Research and Development Program (863) of China under Grant No. 2008AA01Z109, the Key Project of Chinese Ministry of Education under Grant No. 108008, and by the National Key Technology R\&D Program under Grant No. 2006BAK11B00.

\section{References}

1. Eichenberger, A.J., O’Brien, J.K., O’Brien, K.M., Wu, P., et al.: Using advanced compiler technology to exploit the performance of the Cell Broadband Engine architecture. J. IBM Sys. 45, 59-84 (2006)

2. Balart, J., Gonzalez, M., Martorell, X., Ayguade, E., et al.: A Novel Asynchronous Software Cache Implementation for the Cell BE Processor. In: Adve, V., Garzarán, M.J., Petersen, P. (eds.) LCPC 2007. LNCS, vol. 5234, pp. 125-140. Springer, Heidelberg (2008)

3. Lee, J., Seo, S., Kim, C., Kim, J., et al.: COMIC: A Coherent Shared Memory Interface for Cell BE. In: 17th International Conference on Parallel Architectures and Compilation Techniques, pp. 303-314. ACM Press, New York (2008)

4. Marc, G., Nikola, V., Xavier, M., Eduard, A., et al.: Hybrid access-specific software cache techniques for the cell be architecture. In: 17th International Conference on Parallel Architectures and Compilation Techniques, pp. 292-302. ACM, New York (2008)

5. Vujić, N., Gonzàlez, M., Martorell, X., Ayguadé, E.: Automatic Pre-Fetch and Modulo Scheduling Transformations for the Cell BE Architecture. In: Amaral, J.N. (ed.) LCPC 2008. LNCS, vol. 5335, pp. 31-46. Springer, Heidelberg (2008)

6. Chen, T., Lin, H.B., Zhang, T.: Orchestrating data transfer for the cell b.e. processor. In: 22nd Annual International Conference on Supercomputing, pp. 289-298. ACM Press, New York (2008)

7. Dubnicki, C., LeBlanc, T.: Adjustable block size coherent caches. In: 19th Annual International Symposium on Computer Architecture, pp. 170-180. ACM Press, New York (1992)

8. Veidenbaum, A.V., Tang, W., Gupta, R., Nicolau, A., et al.: Adapting Cache Line Size to Application Behavior. In: 13th International Conference on Supercomputing, pp. 145-154. ACM Press, New York (1999) 
9. Seo, S., Lee, J., Sura, Z.: Design and Implementation of Software-Managed Caches for Multicores with Local Memory. In: 15th International Symposium on High-Performance Computer Architecture, pp. 55-66. IEEE Press, New York (2009)

10. Altevogt, P., Boettiger, H., Kiss, T., Krnjajic, Z.: IBM BladeCenter QS21 Hardware Performance. IBM Technical White Paper WP101245 (2008)

11. Bailey, D., Harris, T., Saphir, W., Wijngaart, R.V.D., et al.: The NAS Parallel Benchmarks 2.0. NAS Technical Report NAS-95-020, NASA Ames Research Center, Moffett Field, CA (1995)

12. University of Florida Sparse Matrix Collection, http://www.cise.ufl.edu/research/sparse/matrices/

13. Gelado, I., Kelm, J.H., Ryoo, S., Lumetta, S.S., et al.: CUBA: An Architecture for Efficient CPU/Coprocessor Data Communication. In: 22nd Annual International Conference on Supercomputing, pp. 299-308. ACM Press, New York (2008)

14. Chen, T., Zhang, T., Sura, Z., Tallada, M.G.: Prefetching irregular references for software cache on cell. In: 6th Annual International Symposium on Code Generation and Optimization, pp. 155-164. ACM Press, New York (2008)

15. Cao, Q., Zhao, C.C., Zhang, Y.X., Chen, J.X., et al.: Adaptive Tuning of Sparse MatrixVector Multiplication on Cell Architecture. In: 2nd International Conference on Computer Engineering and Technology, pp. 292-296. IEEE Press, New York (2010)

16. Schneider, S., Yeom, J.S., Rose, B., Linford, J.C., et al.: A Comparison of Programming Models for Multiprocessors with Explicitly Managed Memory Hierarchies. In: 14th ACM SIGPLAN Symposium on Principles and Practice of Parallel Programming, pp. 131-140. ACM, New York (2009)

17. Crawford, C.H., Henning, P., Kistler, M., Wright, C.: Accelerating Computing with the Cell Broadband Engine Processor. In: 5th ACM Conference on Computing Frontiers, pp. 3-12. ACM Press, New York (2008)

18. Wang, Z., O’Boyle, M.: Mapping Parallelism to Multicores: A Machine Learning Based Approach. In: 14th ACM SIGPLAN Symposium on Principles and Practice of Parallel Programming, pp. 75-84. ACM Press, New York (2009)

19. Jimenez, V.J., Vilanova, L., Gelado, I., Gil, M., et al.: Predictive Runtime Code Scheduling for Heterogeneous Architectures. In: Seznec, A., Emer, J.S., O’Boyle, M.F.P., Martonosi, M., Ungerer, T. (eds.) HiPEAC 2009. LNCS, vol. 5409, pp. 19-33. Springer, Heidelberg (2009)

20. Wang, P., Collins, J.D., Chinya, G., Jiang, H., et al.: Architecture and Programming Environment for a Heterogeneous Multicore Multithreaded System. In: Conference on Programming language Design and Implementation, pp. 156-166. ACM, New York (2007)

21. Ren, M., Park, J., Houston, M., Aiken, A., et al.: A tuning framework for softwaremanaged memory hierarchies. In: 17th International Conference on Parallel Architectures and Compilation Techniques, pp. 280-291. ACM, New York (2008) 